\title{
INFLUENCE OF PLANTAR FASCIAL TAPING ON PRESSURE PAIN SENSITIVITY CHANGES IN LOWER EXTREMITY POSTERIOR LINE MUSCLES IN FUTSAL PLAYERS
}

\author{
Sebastian Klich, 1, A, B, C, D Dawid Goliński, , B, D Mateusz Stawarz, 2, B, D Paweł Wolański1, D \\ ${ }^{1}$ Department of Physical Education, Faculty of Physiology and Biochemistry, University School of Physical Education in Wrocław, \\ Wroclaw, Poland \\ 2 Department of Physiotherapy, University School of Physical Education in Wrocław, Wroclaw, Poland

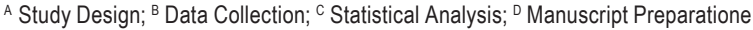 \\ Address for coprespondence: \\ Sebastian Klich, MSc, PT \\ University School of Physical Education in Wrocław, Department of Physical Education, Faculty of Physiology and Biochemistry \\ Paderewskiego Ave 35, 50-612 Wrocław, Poland \\ E-mail: sebastian.klich@gmail.com
}

\begin{abstract}
Ahstract. The aim of the study was to evaluate the threshold of muscle pain sensitivity at the posterior line of the futsal player's lower extremity muscles under the influence of the stimulation of the foot arch with a nonelastic tape application.

The study included 25 futsal players representing university club AZS AWF Wroclaw. During the experiment, a nonelastic tape was applied on the plantar foot surface, basing on the standards of low-Dye taping.The test program consisted of a 3-day cycle, during which the pressure pain threshold (PPT) was measured: 1) before application of the tape, 2) 24 hours and 3) 72 hours after application. The PPT was measured using the Somedic Algometer type 2.

Analysis of the results was performed using analysis of variance for repeated measures and post hoc Bonferroni's test.

Long-term stimulation of plantar surface significantly affects the increase in the PPT in the lower extremity posterior line muscles. In motor control trainings, especially in match-starter terms, new physiotherapeutic measures and therapeutic techniques should be used.
\end{abstract}

Key WOrlls: Low-Dye Taping, prevention, sport injuries, overtraining, futsal

\section{Introduction}

Futsal is a discipline classified as a team game. It is played on a standard handball court, in the sports hall. Furthermore, high dynamism, a lot of take-offs, short distance sprints and changes of running directions constitute futsal and contribute to its spectacularity and popularity (Barbero-Alvarez et al. 2009).

The nascence of muscle overloading in the osteoarticular system constitutes a characteristic movements sequence (e.g. outbursts, feints, running with ball, passing and shots), of the games played on hard surfaces, and depends on the individual predispositions of the player. During the match players engage multiple muscle groups 
that perform mostly a series of eccentric contractions, causing micro-injuries of muscle fibers (Andersen et al. 2006). Intense muscle performance causes muscle soreness, that may be evaluated on the basis of pressure pain threshold (PPT). Muscle soreness may be experienced randomly after the exertion. The individual may be exposed to it during the training session, as well as immediately after it because of the uncontrollable muscle spasms (Nosaka et al. 1991). All of the micro-injuries of the muscle lead to muscle swelling and muscle inflammation. It is manifested by the delayed onset muscle soreness (DOMS), with symptoms such as increased pressure sensitivity (hyperalgesia), rest pain and changes in movement control within the muscle (Woods et al. 2004). Algometry is a method, which allows for the investigation of the muscle sensitivity during squeezing session - one of the main symptoms of DOMS (Jönhagen et al. 2009).

Soreness, strains (including those of in lower extremity posterior line muscles) are one of the most common consequences of the muscle overloading in futsal players. Hamstrings muscle, popliteal muscle and gastrocnemius muscle (including the Achilles tendon) are the most susceptible to injuries. Tension disintegration in the muscles of the lower extremity posterior line may be directly linked to the discordant configuration of the foot insteps, abnormal distribution of the compressive force placed on the plantar foot surface, as well as the inflammation of the plantar fascia. The imbalance between the anterior and posterior muscle groups may be the consequence of those changes (Myers 1997).

All of the harmful effects emerging from the dysfunctions located within the musculoskeletal system may pose a danger in enhancing the skill level of a given player. This constitutes a great challenge for coaches and physiotherapists in the planning of the trainings that are oriented not only towards the development of motor and technical skills, but also on reducing the risk of injuries (Kirkendall and Dvorak 2010).

Futsal is a relatively new team game therefore, it is necessary to deepen the knowledge on the issues related to the prevention of injuries. Further studies will provide the trainers and physiotherapists with an access to a more profound understanding of the issue, which undoubtedly will be reflected in the sport results.

Plantar fascial taping should be used in reducing the plantar fascia tension and arch support, thereby increase of pain sensitivity in muscle groups. In professional sport it is necessary in optimization of overloads. The use of nonelastic tapes on plantar foot surface has been assessed only in a number of studies (Radfort et al. 2006; Russo and Chipehase 2001; Vicenzino et al. 2007). Therefore, the aim of the study was to evaluate the threshold of muscle pain sensitivity at the posterior line of the futsal player's lower extremity muscles under the influence of the stimulation of the foot arch with a nonelastic tape application.

\section{Material and methods}

\section{Participants}

25 male futsal players (age $23.03 \pm 1.15$ years, body height $179.30 \pm 3.56 \mathrm{~cm}$, body weight $76.10 \pm 6.67 \mathrm{~kg}$, BMI $23.40 \pm 2.76 \mathrm{~kg} / \mathrm{m}^{2}$ ) participated in the study. The players were university club AZS AWF Wroclaw members. Training experience of the whole group was on average 14 years. The shortest experience was 8 , and the longest - 17 years of training. The players are the medallists of the Polish Academic Championships in futsal. None of the respondents within the past six weeks has suffered from an injury, none has complained of pain. All subjects were informed about the purpose and the research protocol assumptions. 


\section{Research protocol}

During the experiment, a nonelastic tape was applied on the plantar foot surface, basing on the standards of low-Dye taping. To perform this technique, a nonelastic cotton tape of $4 \mathrm{~cm} \times 5 \mathrm{~m}$ was used. The application was carried out in a supine position with the lower extremity bent at an angle of $90^{\circ}$ in the knee joint. The person applying the tape degreased plantar and dorsal surface of the foot, as well as the medial and lateral edge and, afterwards, the adhesive spray was applied.

Tape application method consisted of 4 stages: 1) bonding the base at a height of 1st to 5th metatarsal head; 2) placing the tape on the medial and lateral edge (start and end of the application at the height of the base); 3) parallel and overlapping application of the tape towards the base (from the base to the calcaneus), and 4) reapplication of the tape at the medial and lateral edge, as well as at the height of metatarsal heads (base) (Figure 1).

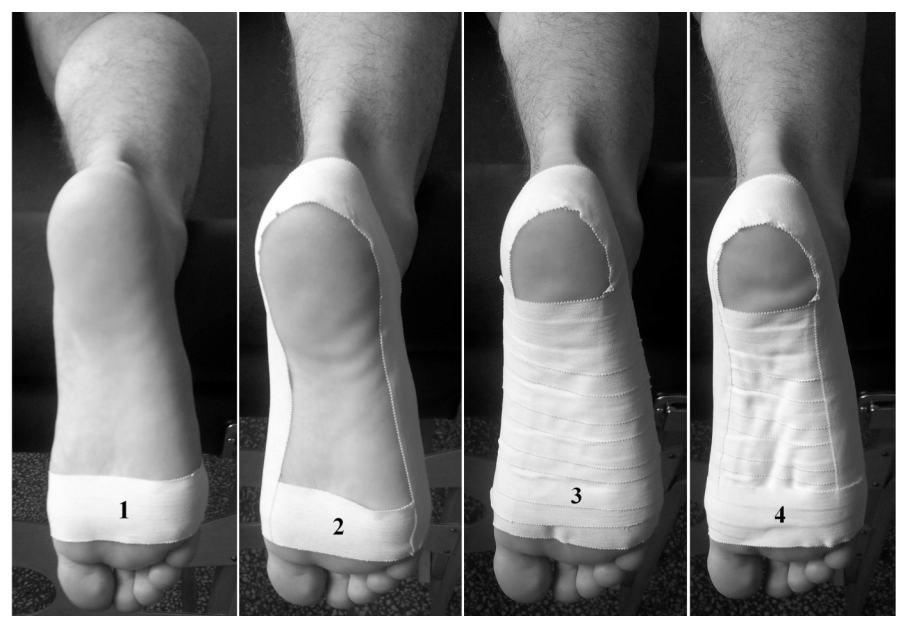

Figure 1. Low-Dye taping technique

The experiment protocol consisted of a 3-day cycle, during which the PPT was measured: 1) before application of the tape, 2), 24 hours and 3) 72 hours after application. PPTs markings were made within the posterior line of the lower extremity muscles. The overall research model assumes that the smaller the value of the PPT, the higher the muscle tenderness.

\section{Research method}

Somedic Algometer type 2 was used to measure the PPTs. The diameter of the tip of the device used for the compression of specific points on the muscle was $10 \mathrm{~mm}$ and covered with $2 \mathrm{~mm}$ of rubber. The pressure on point and its rate was standardized $(30 \mathrm{kPa} / \mathrm{s})$. The value of the pain threshold equals the value of the pressure at which the examined person reported that the pressure exerted on a certain point causes pain (Kawczyński et al. 2013). 
PPT measurements were made on the posterior line of the lower extremity muscles in 13 reference points, ie. (Figure 2): semitendinosus and semimembranosus muscle (points 1-4); biceps femoris muscle (points 5-8); lateral head of the gastrocnemius muscle (points 9, 11); medial head of the gastrocnemius muscle (points 10, 12); and soleus muscle (section 13). Points 1-8 belongs to the posterior thigh muscles (hamstrings muscle), while 9-13 to the posterior shin muscles (gastrocnemius) (Kawczyński et al. 2013).

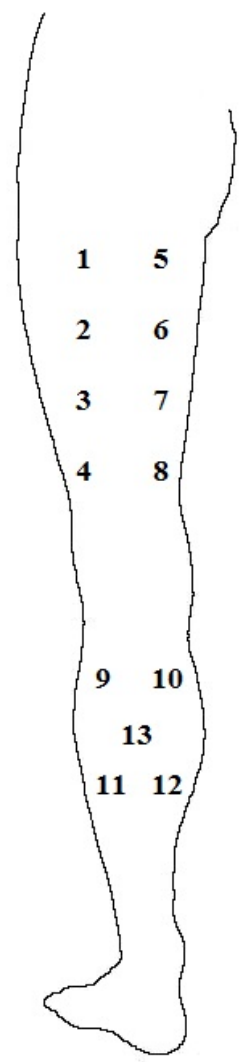

Figule 2. Reference points for PPT assessment

\section{Statistical analysis}

Analysis of the results was performed using the analysis of variance for repeated measures (RMANOVA) and post hoc Bonferroni's test. Before the examination, two tests were conducted to verify the assumptions, namely 1) the normality of distribution - the Shapiro-Wilk test and 2) the homogeneity of variance - Levene's test. Intraclass Correlation Coefficient (ICC) was also assessed in the study and it was $R^{2}=0.912$. Calculations were made using the Statistica $10 \mathrm{PL}$ software. While assessing the differences, it was assumed that they were significant at $p \leq 0.001$. 


\section{Results}

Table 1 shows the complete results of PPT values for all muscle groups, belonging to the lower extremity muscles. Furthermore, the results include reference points of PPT measure (i.e.: 1-8 and 9-13) for futsal players.

Table. 1. Mean values of the PPT and significances before, 24 and 72 hours after the low-Dye Tape application

\begin{tabular}{|c|c|c|c|c|c|c|c|}
\hline \multirow[b]{3}{*}{ Muscle } & \multicolumn{7}{|c|}{ Pressure-pain threshold (PPT) [kPa] } \\
\hline & \multicolumn{3}{|c|}{ right lower extremity } & \multicolumn{3}{|c|}{ left lower extremity } & \multirow[b]{2}{*}{$72 \mathrm{~h}$ after } \\
\hline & reference point & before & $24 \mathrm{~h}$ after & $72 \mathrm{~h}$ after & before & $24 \mathrm{~h}$ after & \\
\hline \multirow{4}{*}{$\begin{array}{l}\text { Semitendinous } \\
\text { Semimembranosus } \\
\text { Muscle }\end{array}$} & 5 & 951.38 & 1125.32 & 1403.29 & 973.15 & 1153.74 & 1475.35 \\
\hline & 6 & 945.76 & 1115.61 & 1448.95 & 934.71 & 1105.63 & 1410.52 \\
\hline & 7 & 942.33 & 1115.37 & 1418.48 & 943.26 & 1117.03 & 1431.44 \\
\hline & 8 & 918.38 & 1114.11 & 1411.52 & 946.81 & 1129.45 & 1443.29 \\
\hline \multirow{4}{*}{ Biceps femoris muscle } & 1 & 909.14 & 1106.45 & 1450.71 & 952.74 & 1150.00 & 1453.50 \\
\hline & 2 & 955.24 & 1146.05 & 1443.71 & 915.14 & 1108.23 & 1407.86 \\
\hline & 3 & 958.38 & 1152.80 & 1435.71 & 936.53 & 1118.48 & 1458.65 \\
\hline & 4 & 978.90 & 1155.06 & 1444.38 & 956.62 & 1142.65 & 1460.81 \\
\hline \multirow{2}{*}{$\begin{array}{l}\text { Lateral head of the } \\
\text { gastrocnemius muscle }\end{array}$} & 9 & 940.33 & 1152.25 & 1452.62 & 952.00 & 1153.79 & 1458.29 \\
\hline & 11 & 948.29 & 1121.68 & 1413.24 & 955.88 & 1130.00 & 1436.00 \\
\hline \multirow{2}{*}{$\begin{array}{l}\text { Medial head of the } \\
\text { gastrocnemius muscle }\end{array}$} & 10 & 943.90 & 1123.40 & 1450.71 & 950.19 & 1131.75 & 1462.38 \\
\hline & 12 & 958.57 & 1173.00 & 1474.29 & 962.59 & 1165.71 & 1466.24 \\
\hline Soleus muscle & 13 & 950.48 & 1134.58 & 1432.57 & 960.86 & 1132.20 & 1436.95 \\
\hline
\end{tabular}

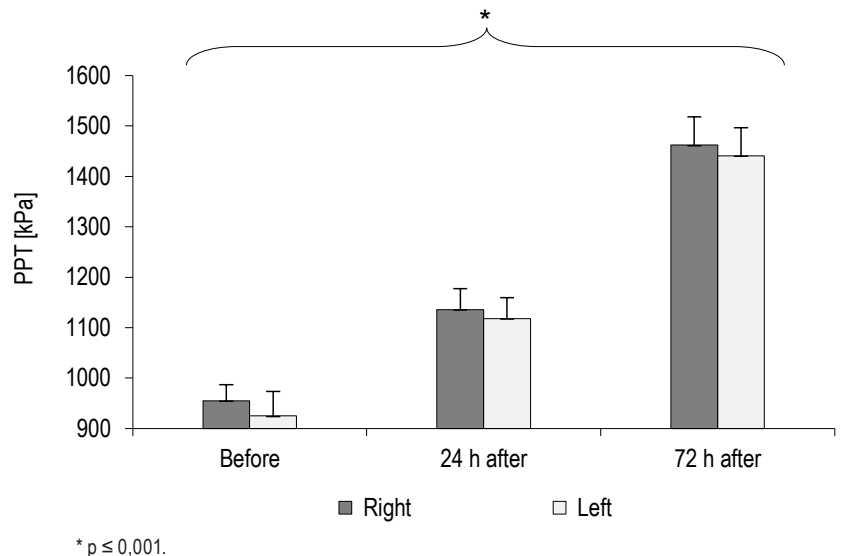

Figule 3. PPT values of thigh muscles before, 24 and 72 hours after taping in futsal players.

The average PPT for posterior thigh muscles in the right lower extremity has significantly changed after 24 and 72 hours after the application (respectively $1135.85 \pm 41.63 \mathrm{kPa} ; \alpha=0.0001 ; 1462.10 \pm 56.13 \mathrm{kPa} ; \mathrm{a}=0.0002$; $p \leq 0.001)$. However, in the initial trial the value was $954.94 \pm 31.63 \mathrm{kPa}$. In comparison with the study before tape application (924.87 $\pm 48.53 \mathrm{kPa}$ ) (Figure 3), a significant increase of PPT was also found in the left lower extremity (respectively $1118.15 \pm 48.50 \mathrm{kPa} ; a=0.0001 ; 1440.68 \pm 55.64 \mathrm{kPa}, a=0.0001 ; p \leq 0.001$ ) (Figure 3). 


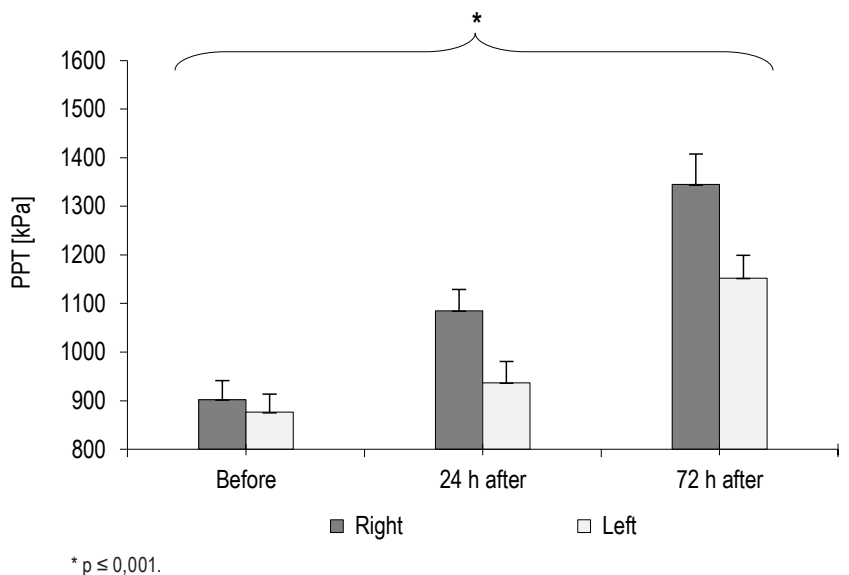

Figulp 4. PPT values of shin muscles before, 24 and 72 hours after taping in futsal players

The PPT value within right lower extremity posterior shin muscles has increased significantly after 24 and 72 hours after the application (respectively $1084.98 \pm 38.84 \mathrm{kPa} ; a=0.0003 ; 1344.69 \pm 62.87 \mathrm{kPa} ; a=0.0002$; $p \leq 0.001)$, in comparison with the initial measure (902.31 $\pm 42.68 \mathrm{kPa})$. While in left lower extremity muscles significant changes after 24 and 72 hours after the application were also observed (respectively $936.69 \pm 44.32 \mathrm{kPa}$; $a=0.001 ; 1151.97 \pm 47.08 \mathrm{kPa} ; a=0.0001 ; p \leq 0.001)$. In the first measurement the value equaled $876.30 \pm 37.46 \mathrm{kPa}$ (Figure 4).

\section{Discussion}

The analysis of the most popular databases of scientific journals proved a limited number of publications relating to futsal. As one of the varieties of football, it is characterized by many interesting aspects, including biomechanical model of postural control, different ergonomics of the game, as well as brand new sets of the factors posing a risk for injuries. Therefore, an attempt to analyse the factors posing a risk for injuries and to verify one of the methods preventing the posterior line of lower extremity muscles has been made.

Epidemiological data relevant to the frequency and the types of the injuries experienced by the futsal players is scarce. This fact can be directly linked to the low popularity of this discipline, as opposed to football (Baroni et al. 2008). A study conducted by Ribeiro et al. (2003), as well as Ribeiro and Pena Costa Ribeiro (2006) proves, that the lower extremities are the segments of the human body, that are the most vulnerable to injuries (constituting about $70 \%$ at whole). The main cause of the injuries of the lower extremity muscles is linked to the reduced mobility (Lentell et al. 1995), muscle flexibility (mainly hamstrings muscle) and the imbalance occurring between the agonistic and antagonistic muscle groups (Knapik et al. 2001; Nasiri and Salehian 2011).

A series of functional changes may be observed in the bodies of futsal players. The impact, that abnormal forces have on the foot, peripheral joins and the higher segments is the main reason behind the occurrence of those functional changes (Ribeiro et al. 2003). The main factor that encourages the emergence of those changes, is the ground, on which the training sessions and matches take place. The most common surface is of hard quality, 
which alters abnormal distribution of pressure on the plantar surface of the foot and overloading located within the ligamental-muscular system (Junge and Dvorak 2010). As a result, the pain formation emerges, which consequently conditions the inflammation, a decrease in flexibility and an increase in the stiffness and muscle tension (Cain et al. 2007). It may be assumed that the overloading and muscle injuries, especially those located in the posterior line of the lower extremity muscles, are caused by the distance covered during the match (Balbero-Alvarez et al. 2008; de Oliviera Bueno et al. 2014), the kinematics of the ball kicks (Barbieri et al. 2010; Ismail et al. 2014), sharp braking and frequent changes of the running direction (Dogramaci et al. 2011).

The results, that have been observed during the study determine a new direction of the research on the reduction of the injury risk. Furthermore, the new therapeutic solutions to the process of physiotherapy are being introduced. The emphasis should be placed on the innovation and novelty that this research provides to the scientific world since there is a scarce and extremely limited number of the studies that engage with the usage of nonelastic taping on the football players in order to prevent the excessive pronation of the foot (Fumich et al. 1981; Sandrey 1996).

The analysis of the data gathered, proved that under the influence of low-Dye taping on the foot, a significant increase in the average value of the PPT may be observed. The PPT value within the hamstrings muscle has increased by $20 \%$ (right) after 24 hours, and by $54 \%$ (right) after 72 hours. Moreover, in the left foot the PPT value within the hamstrings muscle has increased by $21 \%$ after 24 hours, and by $56 \%$ after 72 hours. On the contrary, the increase of the PPT value within the gastrosoleus after 24 hours reached $20 \%$ (right), whereas after 24 hours it indicated $49 \%$ (right). In the left foot an increase of $7 \%$ and $31 \%$ was observed after 24 and 72 hours.

The therapeutic efficiency of the application of low-Dye taping has been confirmed by the examination conducted on the kinematic (Radfort et al. 2006) and electromyographic (Radfort et al. 2006) dynamometric platform (Russo and Chipchase 2001; Vicenzino et al. 2007). However, the studies are engaged with the parts of the body that are located above the foot and ankle. The analysis of the PPT value within the hamstrings muscle and gastrocnemius provides a more profound assessment of the risks of DOMS and injuries (Andersen et al. 2006). The stimulation of the plantar foot surface results in an increase in the stimulus of the plantar fascia, which reduces the excessive tension within the aforementioned structure and the upper segments (Kogler et al. 1999).

The Myers' theory proves, that all structures (muscles, ligaments, and fascia) included in the posterior line are connected with each other. Accordingly, it may be assumed that the alignment/reduction of the excessive tension located within the plantar fascia affects the Achilles tendon, gastrocnemius, and hamstrings muscle (Mayers 1997). This further encourages the occurrence of the new stimuli. Under the influence of the stimulation, the additional stimulation of the skin sensory receptors and proprioceptive sensation occurs, which results in the improvement of the postural control, stability, and balance. The increase in the proprioceptive sensation results in the additional response of the peripheral nervous system, which aims at changing the configuration of a given segment of the motion system and/or equalisation of the motion pattern (Layne et al. 2002).

The aforementioned functional and morphological changes occurring in the control of human movement are the consequence of the reduction of the excessive stiffness, the standardization of tension, increase in the muscle flexibility and changes in the motor unit recruitment strategies (Fromy et al. 2008).

In conclusion, the studies constitute a new path in the discipline of the physiotherapy and the motor preparation of the players. The issue of crucial importance is the continuation of broadening the research by introducing another devices. The studies prepare an interesting ground for further analysis. They may serve in programming and 
monitoring of the trainings throughout the training cycle, as well as, in the monitoring of the regenerative training (e.g. post-match biological regeneration). It should constitute the basic tool in the physiotherapy. What should be highlighted, is the validity of the usage of the aforementioned results in the treatment of the injuries and pain afflictions stemming from the overloading of the musculoskeletal system during the trainings characterised by high intensity and the frequent changes of the surface.

\section{Conclusions}

1. Long-term stimulation of plantar surface significantly affects the increase in the PPT in the lower extremity posterior line muscles.

2. In motor control trainings, especially in match-starter terms new physiotherapeutic measures and therapeutic techniques should be used.

\section{References}

Andersen H., Arendt-Nielsen L., Danneskiold-Samsoe B., Graven-Nielsen T. Pressure pain sensitivity and hardness along human normal and sensitized muscle. Somatosens Mot Res. 2006; 23: 97-109.

Baroni M., Generosi R.A., Pinto Leal Junior E.C. Incidence and factors related to anklesprains in athletes of futsal national teams. Fisioter 2008; 21 (4): 79-88.

Barbero-Alvarez J.C., D'ottavio S., Granda-Vera J., Castagna C. Aerobic fitness in futsal players of different competitive level. J Strength Cond Res, 2009; 23 (7), 2163-2166.

Barbero-Alvarez J.C., Soto VM., Barbero-Alvarez V., Granda-Vera J. Match analysis and heart rate of futsal players during competition. J Sports Sci. 2008; 26 (1): 63-73.

Barbieri F., Gobbi L.B., Santiago P.S., Cunha S. Performance comparisons of the kicking of stationary and rolling balls in a futsal context. Sports Biomechanics. 2010; 9 (1): 1-15.

Cain L.E., Nicholson L.L., Adams R.D., Burns J.. Foot morphology and foot/ankle injury in indoor football. J Sci Med Sport. 2007; 10 (5): 311-319.

De Oliveira Bueno M.J., Caetano F.G., Pereira T.J., De Souza N.M., Moreira G.D, Nakamura F.Y, Cunha S.A, Moura F.A. Analysis of the distance covered by Brazilian professional futsal players during official matches. Sports Biomech. 2014; 13 (3): 230-240.

Dogramaci S.N., Watsford M.L., Murphy A.J. Time-motion analysis of international and national level futsal. J Strength Cond Res. 2011; 25 (3), 646-651.

Fumich R.M., Alison A.E., Querin G.J., Grace P.D. The measured effect of taping on combined foot and ankle motion before and after exercise. Am J Sports Med. 1981; 9 (3): 165-170.

Fromy B., Sigaudo-Roussel D., Saumet J.L. Cutaneous neurovascular interaction involved in tactile sensation. Cardiovasc Hematol Agents Med Chem 2008. 6 (4): 337-342.

Ismail S., Adnan R., Sulaiman N. Moderate Effort Instep Kick in Futsal. Pro Eng. 2014; 72: 186-191.

Jönhagen S., Ackermann P., Saartok T. Forward lunge: a training study of eccentric exercises of the lower limbs. J Strength Cond Res. 2009; 23 (3): 972-978.

Junge A., Dvorak J. Injury risk of playing football in Futsal World Cups. Br J Sport Med. 2010; 44 (15): 1089-1092.

Kawczyński A., Mroczek D., Chmura P., Madeleine P., Chmura J. Influence of a professional football game on delayed onset muscle soreness development In dominant and non-dominant legs. Medycyna Sportowa (Polish J Sport Med). 2013; 29 (1): 55-61.

Kirkendall D.T., Dvorak J. Effective injury prevention in soccer. Phys Sportsmed. 2010; 38 (1): 147-157.

Knapik J.J., Sharp M.A., Canham-Chervak M., Hauret K., Patton J.F., Bruce H.J. Risk factors for training-related injuries among men and women in basic combat training. Med Sci Sports Exerc. 2001; 33: 946-954.

Kogler G.F., Veer F.B., Solomonidis S.E., Paul J.P. The influence of medial and lateral placement of orthotic wedges on loading of the plantar aponeurosis. J Bone Joint Surg Am. 1999; 81 (10): 1403-1413.

Layne C.S., Forth K.E., Baxter M.F., Houser J.J. Voluntary neuromuscular activation is enhanced when paired with a mechanical stimulus to human plantar soles. Neurosci Lett. 2002; 334 (2): 75-78. 
Lentell G., Baas B., Lopez D., Mcgure L., Sarrels M., Snyder P. The contributions of proprioceptive deficits, muscle function, and anatomic laxity to functional instability of the ankle. J Orthop Sports Phys Ther. 1995; 21 (4): 206-215.

Myers T. The anatomy trains. J Bodyw Mov Ther. 1997; 1 (2): 91-101.

Nasiri M., Salehian M.H. Comparison of Flexibility of Pelvic and Femoral Muscles in Futsal, Weightlifting and Swimming. Ann Biologic Res. 2011; 2 (6): 79-83.

Nosaka K., Clarkson P.M., McGuiggin M.E., Byrne J.M. Time course of muscle adaptation after high force eccentric exercise. Eur J Appl Physiol Occup Physiol. 1991; 63: 70-76.

Radford J.A., Burns J., Buchbinder R., Landorf K.B., Cook C. The effect of low-dye taping on kinematic, kinetic, and electromyographic variables: a systematic review. JOSPT. 2006; 36 (4): 232-241.

Ribeiro C.Z.P., Akashi P.M.H., Sacco I.C.N., Pedrinelli A. Relationship between postural changes and injuries of the locomotor system in indoor soccer athletes. Rev Bras Med. Esporte. 2003; 9 (2): 98-103.

Ribeiro R.N., Pena Costa L.O. Epidemiologic analysis of injuries occurred during the 15th Brazilian Indoor Soccer (Futsal) Sub20 Team Selection Championship. Rev Bras Med. Esporte. 2006; 12 (1): 1-5.

Russo S.J., Chipchase L.S. The effect of low-Dye taping on peak plantar pressures of normal feet during gait. Aust J Physiother. 2001; 47 (4): 239-244.

Sandrey M.A. Prevention of Injuries in Excessive Pronators Through Proper Soccer Shoe Fit. J Athl Train. 1996; 31 (3): $231-234$.

Vicenzino B., McPoil T., Buckland S. Plantar Foot Pressures After the Augmented Low Dye Taping Technique. J Athl Train. $2007 ; 42$ (3): 374-380.

Woods C., Hawkins R.D., Maltby S., Hulse M., Thomas A., Hodson A. The Football Association Medical Research Programme: an audit of injuries in professional football - analysis of hamstring injuries. Br J Sports Med. 2004; 38: 36-41.

Cite this article aS." Klich S., Goliński D., Stawarz M., Wolański P. Influence of Plantar Fascial Taping on Pressure Pain Sensitivity Changes in Lower Extremity Posterior Line Muscles in Futsal Players. Central European Journal of Sport Sciences and Medicine. 2015; 11 (3): 115-123. 
